\title{
Avaliação da eficácia da facectomia com implante de lente intra-ocular na infância
}

\author{
Efficacy of pediatric cataract extraction with intraocular lensimplantation
}

\author{
Núbia Cristina de Freitas Maia ${ }^{1}$ \\ Ana Luisa Höfling' Lima ${ }^{2}$ \\ George Baikoff ${ }^{3}$
}

\section{RESUMO}

Objetivo: Avaliar eficácia da utilização da lente intra-ocular na correção da afacia na infância, segundo a acuidade visual e alteração refracional no pós-operatório. Métodos: Foram estudados trinta e três olhos de 27 crianças portadores de catarata, unilateral ou bilateral, submetidos à cirurgia de lensectomia via pars plana com implante de lente intra-ocular, associada à capsulectomia posterior primária e vitrectomia anterior. $\mathrm{O}$ cálculo da lente intra-ocular foi realizado com o objetivo da emetropia no pós-operatório imediato. As crianças apresentavam idades inferiores a seis anos no momento da cirurgia e foram acompanhadas em média durante 2,9 anos. Foram divididas em três grupos: crianças portadoras de catarata unilateral operadas com idade inferior a três anos (Grupo I) e superior a três anos (Grupo II) e grupo III formado pelas crianças portadoras de cataratas bilaterais. Resultados: Acuidade visual pósoperatória obtida no último controle igual ou superior a 20/40 foi encontrada em $85 \%$ dos olhos operados. Equivalente esférico no primeiro mês pós-operatório próximo da emetropia foi obtido em $70 \%$ das crianças do grupo III e em apenas 30\% do grupo I. Quanto à variação refracional pósoperatória, a miopização foi encontrada em $81,81 \%$ dos casos, sendo maior esta alteração refracional quanto menor a idade em que a criança foi submetida à cirurgia. Conclusão: Apesar da miopização pós-operatória que ocorre com o implante de lente intra-ocular na correção da afacia em crianças com menos de seis anos de idade, o resultado visual é bastante satisfatório e a correção refracional residual de fácil execução. Um seguimento pós-operatório mais prolongado há de ser necessário para o acompanhamento dos resultados a longo prazo.

Descritores: Afacia pós-catarata; Catarata/congênito; Lentes intra-oculares; Extração de catarata

\section{INTRODUĈ̃̃O}

A correção da afacia após a cirurgia de catarata na infância depende da idade da criança, do aspecto sócio-familiar e das características próprias do globo ocular. A cirurgia precoce e a adequação da correção óptica são fatores essenciais para um prognóstico funcional favorável ${ }^{(1)}$.

O tratamento da catarata na infância tem evoluído gradativamente, na busca de um procedimento mais seguro e eficiente no tratamento da ambliopia causada pela catarata na infância e com menor taxa de complicações ${ }^{(2)}$. No passado, a correção da afacia congênita era feita exclusivamente com o uso de lentes de contato ou óculos. Ocorrendo a variação refracional, relacionado ao crescimento do globo ocular, esses recursos ópticos eram 
facilmente ajustados. Entretanto, o implante de lente intraocular para a correção da afacia em crianças, está sendo cada vez mais preconizado e aceito pelos especialistas da área ${ }^{(3-6)}$.

O implante de lente intra-ocular em crianças foi efetuado pela primeira vez por Choyce, em 1956, utilizando uma lente de câmara $\operatorname{anterior}^{(7)}$. Foi seguido por Binkhorst, em 1964, que utilizou uma lente de fixação iriana ${ }^{(8)}$. Com o advento das lentes de câmara posterior, o índice de complicações foi bem inferior e o seu uso na correção da afacia em crianças foi mais aceitável ${ }^{(9-10)}$.

Uma das mais cruciais perguntas referente ao implante da lente intra-ocular é o poder dióptrico da lente a ser implanta$\mathrm{da}^{(9,11)}$. Alguns autores preconizam o implante de uma lente com valor dióptrico calculado para emetropia na ocasião da cirurgia $^{(12)}$ ou hipocorrigir com o objetivo de antecipar as mudanças do valor biométrico devido ao crescimento axial do globo ocular ${ }^{(13-16)}$.

Se o olho da criança cresce após o implante da lente intraocular, o erro refrativo resultante pode ser corrigido com lentes de contato, óculos, cirurgia refrativa, como a ceratectomia fotorrefrativa ou até mesmo a troca da lente intra-ocular ${ }^{(17-19)}$.

Para considerar um procedimento cirúrgico viável e seguro, deve-se dar especial atenção aos resultados visuais e à incidência de complicações. O presente estudo tem por objetivo avaliar a eficácia do implante primário da lente intra-ocular de câmara posterior para a correção da afacia na infância, em portadores de catarata de desenvolvimento ou congênita, submetidos à cirurgia em uma idade inferior a 6 anos, utilizando a técnica de lensectomia associada à capsulectomia posterior primária e vitrectomia anterior, segundo a acuidade visual e alteração refracional pós-operatória.

\section{MÉTODOS}

Os olhos operados neste estudo foram considerados como catarata na infância, englobando cataratas congênitas (cataratas com aparecimento nos primeiros três meses de vida) e de desenvolvimento (cataratas que surgiram após os três meses de vida).

Foram inclusos pacientes portadores de catarata congênita ou de desenvolvimento unilateral ou bilateral, de causa idiopática ou hereditária, com grau de densidade de opacificação do cristalino variável que impedia o exame retinoscópico ou fundoscópico. Foram excluídos os pacientes portadores de cataratas de causa infecciosa, secundárias a erros inatos do metabolismo ou distúrbios genéticos e crianças portadoras de doenças sistêmicas ou outras lesões oculares associadas (microftalmia, alterações pupilares, nistagmo, alterações do segmento anterior, comprometimento do segmento posterior).

Todas as crianças foram examinadas no pré-operatório pelo mesmo médico. O exame oftalmológico completo compreendia testes de avaliação da acuidade visual (através de cartões visuais de Teller ou tabela de Snellen), pesquisa dos reflexos pupilares, biomicroscopia do segmento anterior, exame da retina com oftalmoscópio indireto, retinoscopia sob cicloplegia. O exame sob narcose era realizado em crianças muito pequenas com dificuldade de colaboração.

Nos casos em que não era possível a execução do exame da retina com oftalmoscópio indireto realizava-se a ultra-sonografia do olho, pelos modos A e B, sendo possível assim a verificação de alterações nas estruturas intra-oculares e o posicionamento da retina.

A técnica cirúrgica para remoção do cristalino utilizada em todas as crianças, foi a lensectomia via pars plana, vitrectomia do terço anterior do vítreo, capsulectomia anterior e posterior, e implante primário de lente intra-ocular de câmara posterior. Todas as cirurgias foram realizadas pelo mesmo cirurgião (G.B.), na clínica Monticelli, em Marseille, França.

A dioptria da lente intra-ocular utilizada em cada caso foi calculada para a obtenção de emetropia em todas as crianças dos grupos estudados, através do programa de regressão linear de Sanders, Retzlaff e Kraff II (SRK II) ${ }^{(20)}$. As crianças com boa colaboração puderam ter a ceratometria avaliada utilizando-se um ceratômetro manual ou computadorizado e a ecobiometria realizada sem narcose. Em algumas crianças com dificuldade de colaborar, a medida da ceratometria não foi confiável, sendo portanto utilizada uma ceratometria média de 45,00D e a ecobiometria realizada sob narcose.

Todos os implantes de lente intra-oculares utilizados foram de peça única e sem furos de manipulação, de polimetilmetacrilato (PMMA), de diversos fabricantes e escolhidos de acordo com a disponibilidade dos mesmos no bloco cirúrgico onde foram realizadas as cirurgias.

No primeiro mês do período pós-operatório, os retornos para controle foram semanais. A partir do segundo mês até o quarto mês, os retornos foram mensais. Após o sexto mês, os retornos foram anuais. Algumas famílias procedentes de outras regiões foram orientadas a manter os retornos periódicos com os médicos oftalmologistas clínicos da sua localidade.

A acuidade visual corrigida pós-operatória foi aferida utilizando armação de prova adequada e a tabela de Snellen ou os cartões visuais de Teller.

A acuidade visual foi analisada fazendo a conversão da mesma em $\log$ MAR. A conversão foi feita calculando o valor negativo do logarítimo do número decimal da acuidade visual obtida pela tabela de Snellen ou pelos cartões visuais de Teller ${ }^{(21)}$.

Para análise da evolução da acuidade visual, foi calculado a média em logMAR de todas as acuidades visuais obtidas de cada paciente nas visitas em cada período de tempo, assim distribuídos: Intervalo 1 (0-1 ano), Intervalo 2 (1-2 anos), Intervalo 3 (2-3 anos) e Intervalo 4 (>3 anos)

A avaliação refracional foi realizada com o exame de retinoscopia em todos os casos, e nas crianças colaborativas foi também utilizado a refratometria computadorizada. Os resultados da retinoscopia ou da refratometria computadorizada em dioptrias, obtidos para longe, foram convertidos em equivalente esférico para a análise neste estudo. Foi considerada como primeira refração confiável, a refratometria realizada na visita com 1 mês de pós-operatório. 
A variação refracional ao longo de todo seguimento pósoperatório foi analisada utilizando a média de todos os equivalentes esféricos de cada paciente, obtida nas visitas em cada período de tempo, assim distribuídos: Intervalo 1 (0-1 ano), Intervalo 2 (1-2 anos), Intervalo 3 (2-3 anos) e Intervalo 4 (>3 anos).

Em todos os casos, o nível de rejeição para a hipótese de nulidade foi fixado em um valor menor ou igual a $0,05(5 \%)$.

\section{RESULTADOS}

Foram avaliados 33 olhos em 27 crianças (17 do sexo masculino e 10 do sexo feminino) com menos de seis anos de idade. Para análise dos dados, foram separados em três grupos: grupo I formado pelas crianças portadoras de cataratas unilaterais operadas com menos de três anos de idade (10 crianças), grupo II pelas crianças com cataratas unilaterais operadas com idade superior a 3 anos (11 crianças), e grupo III, pelas crianças portadoras de cataratas bilaterais (6 crianças). O seguimento pós-operatório variou de um a seis anos, com média de 2,9 anos. Foram realizadas no mínimo 3 visitas com refração confiável para cada olho.

Neste estudo, o valor da lente a ser implantado foi calculado para obter a emetropia e o poder dióptrico da lente implantada foi em média de 25,55 D no grupo I; 25,23D no grupo II e 26,42D no grupo III. Não foi encontrada diferença estatisticamente significante quanto ao poder da lente. $\mathrm{Na}$ análise dos erros biométricos encontrados no primeiro mês pós-operatório nos grupos estudados, observa-se que aproximadamente $50 \%$ das crianças obtiveram um equivalente esférico com erro até 1D. A mediana dos erros de cálculo do poder da lente intra-ocular implantada no grupo I foi maior que no grupo III, e esta diferença foi estatisticamente significante $(\mathrm{p}=0,015)$ (Tabela 1$)$.
Todos os grupos avaliados tiveram uma tendência de miopização ao longo do tempo, ou seja, na última visita os olhos estavam mais míopes ou menos hipermétropes que a refração inicial, sendo estatisticamente significativa esta variação refracional nos grupo I e III ( $\mathrm{p}<0,001)$ (Tabela 2).

$\mathrm{Na}$ avaliação da dependência da miopização com a variável idade e poder dióptrico somente a variável idade foi estatisticamente importante como preditora de acordo com a regressão linear múltipla por passos lógicos (Gráfico 1). Assim sendo, o modelo utilizado foi o de regressão simples. A variável idade influenciou na miopização de forma discreta $(15,8 \%)$, porém de forma estatisticamente significativa $(\mathrm{p}=0,0402)$.

$\mathrm{Na}$ análise da média da melhor acuidade visual obtida na última visita do controle pós-operatório, não se encontrou diferença estatisticamente significante entre os grupos $(\mathrm{p}=0,2563)$, embora não houvesse poder de teste suficiente para afirmar que as diferenças realmente não existem $($ Poder $=0,1)$, pois a amostra é pequena (Gráfico 2).

$\mathrm{Na}$ análise da média da acuidade visual ao longo do seguimento pós-operatório, observa-se que há uma tendência que cada grupo apresente uma evolução distinta ao longo do tempo, porém esta indicação não foi estatisticamente significante $(\mathrm{p}=0,054)($ Gráfico 3$)$.

\section{DISCUSSÃO}

Uma criança que necessitou de uma extração de cristalino, decorrente de uma opacidade, seja ela por motivo congênito ou adquirido, necessita de uma correção da sua hipermetropia para ver claramente ${ }^{(6)}$

O uso de lentes intra-oculares em crianças vem se tornando cada vez mais comum, resultando numa correção da hipermetropia de forma constante e duradoura ${ }^{(10,22)}$.

\begin{tabular}{|c|c|c|c|c|c|c|c|c|}
\hline \multirow{2}{*}{$\begin{array}{l}\text { Variação em } \\
\text { dioptrias }\end{array}$} & \multicolumn{2}{|c|}{ Grupo I } & \multicolumn{2}{|c|}{ Grupo II } & \multicolumn{2}{|c|}{ Grupo III } & \multicolumn{2}{|c|}{ Total } \\
\hline & $\mathbf{n}$ & $\%$ & $\mathbf{n}$ & $\%$ & $\mathbf{n}$ & $\%$ & $n$ & $\%$ \\
\hline Até 1D & 3 & 30,00 & 5 & 45,45 & 8 & 66,66 & 16 & 48,48 \\
\hline$>3 D$ & 4 & 40,00 & 1 & 9,10 & 2 & 16,66 & 7 & 21,21 \\
\hline Total & 10 & 100,00 & 11 & 100,00 & 12 & 100,00 & 33 & 100,00 \\
\hline
\end{tabular}

\begin{tabular}{|c|c|c|c|c|c|c|c|c|}
\hline \multirow[t]{2}{*}{ Grupos } & \multicolumn{8}{|c|}{ Média dos equivalentes esféricos } \\
\hline & RX 1 & $n$ & RX 2 & $n$ & RX 3 & $n$ & RX 4 & $n$ \\
\hline Grupo II & $-0,74$ & 11 & $-1,40$ & 9 & $-2,22$ & 9 & $-2,85$ & 5 \\
\hline Grupo III* & 0,37 & 12 & 0,33 & 11 & $-0,13$ & 12 & $-0,69$ & 8 \\
\hline \multicolumn{9}{|c|}{$\begin{array}{l}\text { Análise de variância com medidas repetidas; } n=\text { número de casos; } R X 1=\text { média dos equivalentes esféricos obtida no intervalo de tempo } 1(0-1 \text { ano); RX } 2=\text { média } \\
\text { dos equivalentes esféricos obtida no intervalo de tempo } 2(1-2 \text { anos); } R X 3=\text { média dos equivalentes esféricos obtida no intervalo de tempo } 3 \text { ( } 2-3 \text { anos); RX } 4=\text { média } \\
\text { dos equivalentes esféricos obtida no intervalo de tempo } 4 \text { ( }>3 \text { anos) } \\
\text { "estatisticamente significante }(p<0,001)\end{array}$} \\
\hline
\end{tabular}




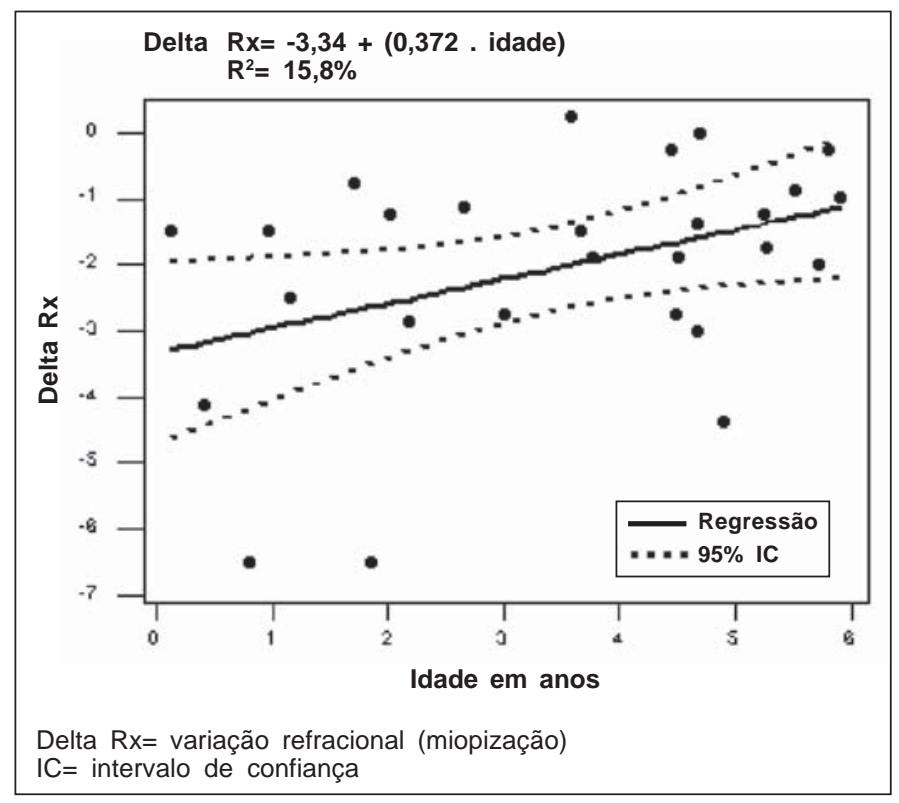

Gráfico 1 - Regressão linear da variação refracional, segundo a idade no momento da cirurgia, em um seguimento pós-operatório médio de 2,9 anos nos grupos de portadores de catarata na infância submetidos a cirurgia

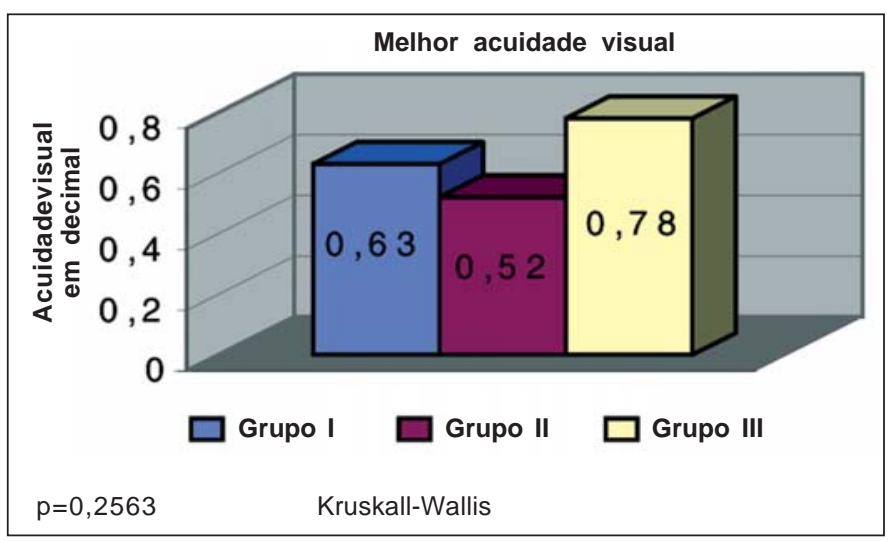

Gráfico 2 - Distribuição da média da melhor acuidade visual obtida no último controle, nos grupos estudados

Em relação ao poder da lente a ser implantada em crianças, dois raciocínios podem ser usados: o implante de uma lente com objetivo de emetropização no pós-operatório imediato ou produzir um erro refrativo inicial de hipermetropia, considerando-se que ocorrerá uma diminuição desta hipermetropia ao longo do pós-operatório ${ }^{(11-12,14,23-24)}$.

Alguns autores preconizam um desconto de 6 dioptrias do poder dióptrico da lente calculada para emetropia em olhos operados entre 4 e 8 semanas e de 3 dioptrias em crianças de 1 a 4 anos de idade. Em crianças entre 5 e 12 anos de idade, a refração inicial desejada seria de 1 dioptria de hipermetropia. Ao longo do acompanhamento a correção com óculos seria atualizada de acordo com a miopização pós-operatória ${ }^{(24)}$.

Em um estudo de uma série de 21 olhos pseudofácicos,

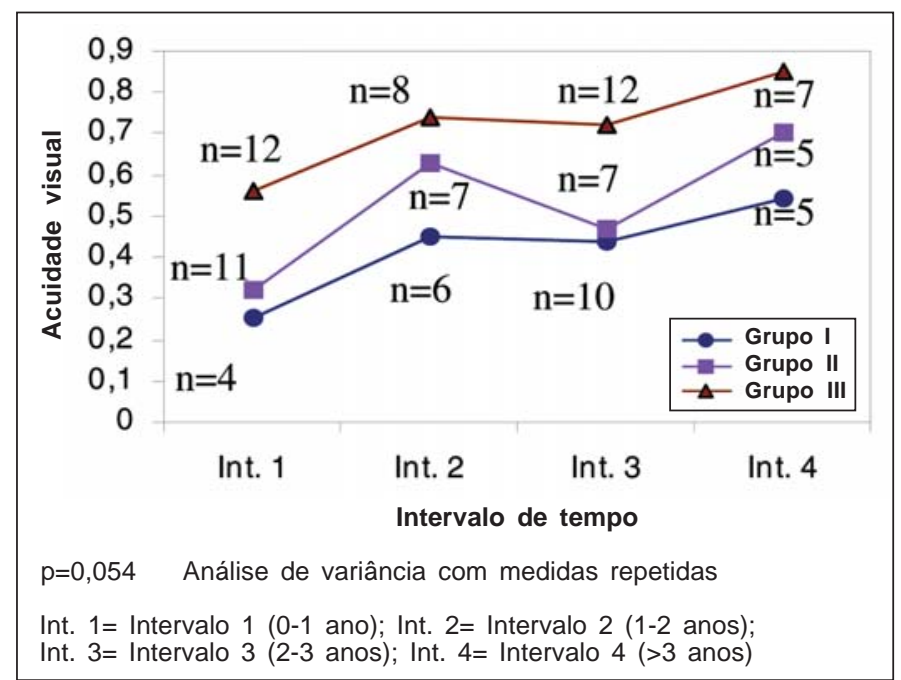

Gráfico 3 - Evolução da média da acuidade visual corrigida nos diversos grupos, ao longo do tempo de seguimento pós-operatório

submetidos à cirurgia entre 4 e 8 meses de idade, os autores recomendam uma hipocorreção de $60 \%$ dos valor dióptrico da lente calculada pela fórmula SRK II ${ }^{(23)}$.

Em um outro estudo que compreende 156 olhos pseudofácicos operados de catarata entre 1 mês e 8 anos de idade, os autores recomendam uma hipocorreção de $20 \%$ do valor da lente a ser implantada no grupo de crianças operadas entre 1 mês e 18 meses de idade, mas neste caso, utilizando uma ceratometria média de $44 \mathrm{D}^{(25)}$.

Outros autores sugerem uma hipocorreção de 35\% do valor dióptrico da lente intra-ocular para crianças menores de 6 meses e $25 \%$ para crianças entre 7 e 11 meses $^{(26)}$.

Implantar lentes intra-oculares objetivando a emetropia da criança no momento da cirurgia, diminuindo assim a sua dependência dos recursos ópticos nos primeiros anos de seguimento pós-operatório, é também uma forma correta de correção da afacia infantil ${ }^{(12)}$.

Todas as lentes implantadas neste estudo foram calculadas com o objetivo da emetropia no momento da cirurgia. Devido às dificuldades no cálculo do poder dióptrico e das dificuldades técnicas inerentes para a ecobiometria ocular em crianças, este objetivo só foi alcançado em 1 olho do grupo das cataratas unilaterais maiores que 3 anos e 3 olhos no grupo de cataratas bilaterais.

Aproximadamente 50\% dos olhos operados apresentavam um erro refrativo inicial de até 1D. No grupo das cataratas unilaterais menores que 3 anos de idade, $40 \%$ dos olhos operados tinham um erro biométrico acima de 3D. Nos grupos de catarata acima de 3 anos (unilaterais e bilaterais), aproximadamente $90 \%$ dos olhos operados apresentavam um erro refrativo inicial menor que 3D.

Neste estudo, a maior mediana de erros do cálculo do poder da lente intra-ocular implantada foi encontrada no grupo das cataratas unilaterais menores que três anos, sendo estatisticamente significante. Isto pode ser explicado porque a ceratome- 
tria corneana utilizada na fórmula para o cálculo da lente neste grupo de crianças não colaborativas foi uma ceratometria média, devido à dificuldade de mensuração. Nas crianças colaborativas maiores que 3 anos foi possível avaliar a ceratometria corneana, permitindo assim uma maior precisão no cálculo do poder dióptrico da lente. Os maiores erros de cálculo da lente intra-ocular nas crianças pequenas também podem ter sido causados por erros inerentes à própria fórmula que podem ocorrer quando o diâmetro axial é muito pequeno ${ }^{(12,20,27)}$.

As dificuldades de mensurar com precisão a ceratometria corneana de crianças muito pequenas podem levar a erros de até 6 dioptrias na refração final pós-operatória desejada, mesmo tendo em mãos ceratômetros manuais e realizando exames sob anestesia geral $^{(28)}$.

Quanto à variação refracional não há muitos estudos de longo seguimento pós-operatório em crianças pseudofácicas, mas mudanças refracionais maiores que $10 \mathrm{D}$ já foram relatadas ${ }^{(29)}$.

A variação refracional denominada miopização pós-operatória é quando ocorre um decréscimo ao longo do tempo da hipermetropia inicial ou um aumento progressivo da miopia, nesse caso caracterizado como miopia axial, decorrente do crescimento antero-posterior do globo ocular ${ }^{(6,29,30)}$. Em um estudo do efeito da implantação de lentes intra-oculares em crianças, os autores observaram uma miopização pós-operatória em $86,7 \%$ dos olhos operados com máxima de $11 \mathrm{D}^{(30)}$.

Neste estudo, observou uma tendência de miopização pósoperatória em $81,81 \%$ dos olhos operados ao longo de todo o tempo de seguimento pós-operatório. Todos os grupos apresentaram uma tendência à miopização, sendo estatisticamente significante as variações refracionais observadas no grupo de cataratas unilaterais menores que 3 anos e no grupo de cataratas bilaterais. Ou seja, na avaliação das médias dos equivalentes esféricos obtidos nos períodos de tempo pós-operatório $\left(1^{\circ}\right.$ ano, $2^{\circ}$ ano, $3^{\circ}$ ano e acima de 4 anos), a média do equivalente esférico encontrada no último intervalo de tempo (acima de 4 anos) era significantemente mais míope que a média da refração inicial obtida no primeiro ano de seguimento pós-operatório.

A variável idade foi a única variável estatisticamente importante como preditora da miopização, ao longo de todo o seguimento pós-operatório. A idade influenciou na miopização discretamente, mas de forma estatisticamente significativa $(\mathrm{p}=0,0402)$. Observamos que quanto menor a idade em que a criança foi submetida à cirurgia, maior a miopização no seguimento pós-operatório. Pela fórmula da variação refracional, uma criança de 1 ano de idade teria uma miopização de aproximadamente 3 dioptrias em um seguimento pós-operatório de 2,9 anos. Uma criança de 3 anos, durante o mesmo tempo de pós-operatório, teria uma miopização de 2,22 dioptrias.

Em um estudo onde foram implantadas lentes intra-oculares em crianças menores que 1 ano de idade, os autores observaram uma média de 6 dioptrias de miopização pós-operatória nos 41 meses de seguimento ${ }^{(31)}$.

Através de um seguimento pós-operatório de 3 anos de 52 olhos operados, os autores observaram uma miopização de 5,96 dioptrias em crianças operadas com idade inferior a 2 anos. Crianças entre 3 e 4 anos tiveram uma miopização de 3,66 dioptrias e crianças entre 5 e 6 anos tiveram uma miopização de 3,40 dioptrias $^{(32)}$.

Quando uma catarata é removida em criança e é realizado o implante da lente intra-ocular, a natureza estática do poder dióptrico da lente resulta em uma maior miopização com o crescimento do olho. Esta miopização pode ser magnificada pelo poder dióptrico da lente, um fenômeno óptico que pode ser compreendido pelo distanciamento da lente intra-ocular em relação ao plano retiniano, decorrente do crescimento do diâmetro ântero-posterior, simulando ao efeito da distância vértice em lentes de alto poder dióptrico ${ }^{(29)}$.

Neste estudo, o poder dióptrico da lente implantada em crianças menores de três anos de idade com catarata unilateral foi em média de 25,55 D. Nas crianças maiores de 3 anos de idade, o poder foi de 25,23 e 26,42 nos grupos de catarata unilateral e bilateral respectivamente. Não foi encontrada diferença estatisticamente significante quanto ao poder da lente, o que não seria esperado, uma vez que as crianças menores que três anos apresentam um diâmetro axial menor quando comparado com crianças maiores que três anos e portanto, mais hipermétropes, necessitando de lentes intra-oculares de maiores poderes dióptricos. No entanto, as crianças menores que três anos neste estudo não foram colaborativas para a avaliação da ceratometria e a chance de erro no cálculo do poder dióptrico da lente intra-ocular foi maior. Uma vez que os poderes dióptricos não diferiram entre os grupos, provavelmente isto explica o fato de que apenas a idade foi um fator preditor da miopização, excluindo a influência do poder dióptrico da lente implantada.

O resultado visual de olhos operados de catarata congênita depende da idade de início da catarata, da idade em que a cirurgia foi realizada, do tipo morfológico da catarata e o acompanhamento e tratamento da ambliopia ${ }^{(2)}$. Analisando a literatura sobre catarata congênita, considera-se sucesso no tratamento quando a acuidade visual final obtida é igual ou maior que 20/40 ${ }^{(33)}$.

Em um estudo brasileiro onde foram avaliadas as acuidades visuais dos olhos pseudofácicos em crianças portadoras de catarata bilateral, os autores encontraram uma taxa de sucesso de $48 \%$. Estas crianças foram operadas numa faixa etária entre 4 e 10 anos de idade ${ }^{(34)}$.

Neste estudo, a taxa de sucesso no grupo de catarata unilateral menor que 3 anos foi de $70 \%$, nos olhos com catarata unilateral maior que 3 anos foi de $81 \%$, e nos olhos com catarata bilateral foi de $100 \%$. Não houve diferença estatisticamente significante entre a melhor acuidade visual corrigida obtida no último controle entre os grupos, no entanto não houve poder de teste suficiente para dizer que as diferenças realmente não existem. Há uma tendência que cada grupo apresente uma evolução distinta ao longo do tempo, porém esta indicação não foi estatisticamente significante $(\mathrm{p}=0,054)$. Pelo gráfico o grupo de cataratas bilaterais teria uma evolução visual mais favorável que o grupo de cataratas unilaterais. Acredita-se que o pequeno número de olhos em cada momento pós-operatório tenha prejudicado a capacidade de análise 
(análise de variância com medidas repetidas). Ao analisar os indivíduos sem considerar os grupos, perdemos a significância ao longo do tempo.

Numa análise de 52 olhos com catarata congênita submetidos à cirurgia, os autores observaram melhores resultados visuais no grupo das cataratas bilaterais, obtendo $95 \%$ com acuidade visual igual ou superior a 20/30. No grupo das cataratas unilaterais, $74 \%$ dos olhos obtiveram acuidade visual igual ou superior a $20 / 50^{(32)}$.

O tipo morfológico da catarata pode influenciar no resultado visual final. Catarata total presente ao nascimento e polar posterior são os tipos que causam maior ambliopia, quando não operadas precocemente. Cataratas unilaterais têm piores resultados, quando comparados com cataratas bilaterais ${ }^{(35)}$. O excelente resultado encontrado neste estudo pode ser explicado pelo fato que a grande maioria das crianças apresentava catarata zonular que são menos ambliogênicas (73\%). Além deste fato, nenhuma criança apresentava nistagmo pré-operatório ou outra anomalia associada.

A ausência de nistagmo pode indicar que ou a visão era melhor que 20/200, ou que a opacidade tornou-se mais densa após o período crítico de fixação. Cataratas congênitas não associadas com outras anomalias oculares tendem a ter resultados visuais melhores ${ }^{(35)}$.

Outro fator a ser considerado é que a miopização pós-operatória não impediu os bons resultados visuais, desde que devidamente corrigidos com óculos ao longo do acompanhamento pósoperatório. É conhecido que anisometropias miópicas são menos ambliogênicas que as anisometropias hipermetrópicas ${ }^{(36)}$.

A correção da afacia infantil com lente intra-ocular apresenta-se como uma boa alternativa de tratamento, permitindo uma correção permanente e melhorando a dependência de outros recursos ópticos para as altas hipermetropias. Calcular o valor da lente a ser implantada é um grande desafio para todo cirurgião, entretanto o implante de uma lente calculada para emetropia e a adequação da correção refracional da miopização ao longo do tempo pós-operatório não impedem os bons resultados visuais. No entanto, ainda serão necessários outros estudos, com um maior tempo de seguimento pós-operatório, para avaliarmos com maior segurança sua aplicabilidade.

\section{ABSTRACT}

Purpose: To evaluate the efficacy of the use of intraocular lenses in the treatment of pediatric aphakia, according to postoperative visual acuity and refraction change. Methods: A total of 33 eyes in 27 children were studied. Children with either unilateral or bilateral cataracts were submitted to lensectomy surgery via pars plana, with intraocular lens implant, associated with primary posterior capsulectomy and anterior vitrectomy. The intraocular lenses were calculated for emmetropia in the first postoperative month. All children were less than six years old at the time of the surgery and had a 2.9 year average follow-up. They were divided into 3 groups. Group I
(10 eyes), children with unilateral cataracts and under three years old at the time of the surgery; group II (11 eyes), children with unilateral cataracts and above three years old; group III (12 eyes), children with bilateral cataracts and above three years old at the time of the surgery. Results: On the last follow-up examination recorded visual acuity was equal to or above $20 / 40$ in $85 \%$ of the eyes. A spherical equivalent close to emmetropia in the first postoperative month was obtained in $70 \%$ of the children of group III but only in $30 \%$ of group I. Regarding postoperative refraction variation, myopic shift was detected in $81.81 \%$ of the cases. The younger the children were when undergoing surgery, the greater the refractional alteration. Conclusion: Despite the myopic shift that happens with the use of intraocular lenses in the treatment of pediatric aphakia in children under six years old, the visual result is very good and the residual refraction correction is easily performed. A more prolonged postoperative follow-up would be necessary for long-term evaluation of the results.

Keywords: Postcataract aphakia; Cataract/congenital; Intraocular lenses; Cataract extraction

\section{REFERÊNCIAS}

1. Moore BD. Optometric management of congenital cataracts. J Am Optom Assoc. 1994;65(10):719-24.

2. Nelson LB. Diagnosis and management of cataracts in infancy and childhood. Ophthalmic Surg. 1984;15(8):688-97.

3. Hiles DA. Intraocular lens implantation in children with monocular cataracts. 1974-1983. Ophthalmology.1984;91(10):1231-7.

4. Dahan E, Salmenson BD. Pseudophakia in children: precautions, technique, and feasibility. J Cataract Refract Surg. 1990;16(1):75-82.

5. Gimbel HV, Ferensowicz M, Raanan M, DeLuca M. Implantation in children. J Pediatr Ophthalmol Strabismus. 1993;30(2):69-79.

6. McClatchey SK, Parks MM. Myopic shift after cataract removal in childhood. J Pediatr Ophthalmol Strabismus. 1997;34(2):88-95.

7. Choyce DP. Correction of monocular aphakia by means of anterior chamber acrylic implants. Trans Ophthalmol Soc U K. 1956;78:459.

8. Binkhorst CD, Gobin MH. Injuries to the Eye with Lens Opacity in Young Children. Ophthalmologica. 1964;148:169-83.

9. Xie L, Dong X, Cao J, Li S, Shi W, Ji H. [Congenital cataract extraction with intraocular lens implantation in children]. Zhonghua Yan Ke Za Zhi. 1998;34(2):99-102.

10. Young TL, Bloom JN, Ruttum M, Sprunger DT, Weinstein JM. The IOLAB, Inc pediatric intraocular lens study. AAPOS Reasearch Committee. American Association for Pediatric Ophthalmology and Strabismus. J Aapos. 1999;3(5):295-302.

11. McClatchey SK. Intraocular lens calculator for childhood cataract. J Cataract Refract Surg. 1998;24(8):1125-9.

12. Brady KM, Atkinson CS, Kilty LA, Hiles DA. Cataract surgery and intraocular lens implantation in children. Am J Ophthalmol. 1995;120(1):1-9.

13. Zetterstrom C, Kugelberg U, Oscarson C. Cataract surgery in children with capsulorhexis of anterior and posterior capsules and heparin-surface-modified intraocular lenses. J Cataract Refract Surg. 1994;20(6):599-601.

14. Lambert SR, Drack AV. Infantile cataracts. Surv Ophthalmol. 1996;40(6):427-58.

15. Cheng KP. Pediatric cataracts. Curr Opin Ophthalmol 1996;7(1):63-8.

16. Lesueur L, Arne JL, Chapotot E. [Predictability of intraocular lens power calculation in the treatment of cataracts in children]. J Fr Ophtalmol. 1999;22 (2):209-12. French.

17. Singh D. Photorefractive keratectomy in pediatric patients. J Cataract Refract Surg. 1995;21(6):630-2.

18. Wilson ME, Peterseim MW, Englert JA, Lall-Trail JK, Elliott LA. Pseudophakia and polypseudophakia in the first year of life. J Aapos. 2001;5(4):238-45.

19. Pavlovic S. [Cataract surgery in children]. Med Pregl. 2000;53(5-6):257-61. 
20. Sanders DR, Retzlaff J, Kraff MC. Comparison of the SRK II formula and other second generation formulas. J Cataract Refract Surg. 1988;14(2):136-41.

21. Holladay JT. Proper method for calculating average visual acuity. J Refract Surg. 1997;13(4):388-91.

22. Malukiewicz-Wisniewska G, Kaluzny J, Lesiewska-Junk H, Eliks I. Intraocular lens implantation in children and youth. J Pediatr Ophthalmol Strabismus. 1999;36(3):129-33.

23. Vasavada A, Chauhan H. Intraocular lens implantation in infants with congenital cataracts. J Cataract Refract Surg. 1994;20(6):592-8.

24. Flitcroft DI, Knight-Nanan D, Bowell R, Lanigan B, O'Keefe M. Intraocular lenses in children: changes in axial length, corneal curvature, and refraction. Br J Ophthalmol. 1999;83(3):265-9.

25. Dahan E, Drusedau MU. Choice of lens and dioptric power in pediatric pseudophakia. J Cataract Refract Surg. 1997;23 Suppl 1:618-23.

26. De Laage De Meux P, Zafad R, Arndt C, Caputo G, Meunier I, Edelson C. [Intraocular lens implantation in the first year of life]. J Fr Ophtalmol. 2001;24(4):360-5. French.

27. Kalogeropoulos C, Aspiotis M, Stefaniotou M, Psilas K. Factors influencing the accuracy of the SRK formula in the intraocular lens power calculation. Doc Ophthalmol. 1994;85(3):223-42.

28. Mittelviefhaus H, Gentner C. [Errors in keratometry for intraocular lens implantation in infants]. Ophthalmologe. 2000;97(3):186-8.
29. McClatchey SK, Dahan E, Maselli E, Gimbel HV, Wilson ME, Lambert SR, et al. A comparison of the rate of refractive growth in pediatric aphakic and pseudophakic eyes. Ophthalmology. 2000;107(1):118-22.

30. Kora Y, Inatomi M, Fukado Y, Marumori M, Yaguchi S. Long-term study of children with implanted intraocular lenses. J Cataract Refract Surg. 1992; 18(5):485-8.

31. O'Keefe M, Fenton S, Lanigan B. Visual outcomes and complications of posterior chamber intraocular lens implantation in the first year of life. J Cataract Refract Surg. 2001;27(12):2006-11.

32. Crouch ER, Crouch Jr ER, Pressman SH. Prospective analysis of pediatric pseudophakia: myopic shift and postoperative outcomes. J Aapos. 2002;6(5): 277-82.

33. Crouch ER, Jr., Pressman SH, Crouch ER. Posterior chamber intraocular lenses: long-term results in pediatric cataract patients. J Pediatr Ophthalmol Strabismus. 1995;32(4):210-8.

34. Tartarella MB, Kawakami LT, Scarpi MJ, Hayashi S, Bonomo PPO. Aspectos cirúrgicos em catarata congênita. Arq Bras Oftalmol. 1995;58(1):24-28.

35. Jain IS, Pillai P, Gangwar DN, Gopal L, Dhir SP. Congenital cataract: management and results. J Pediatr Ophthalmol Strabismus. 1983;20(6):243-6.

36. Weakley DR, Jr. The association between nonstrabismic anisometropia, amblyopia, and subnormal binocularity. Ophthalmology. 2001;108(1):163-71. 